\title{
Pour une didactique de la cruauté. Le savoir-faire de l'écriture chez Agota Kristof
}

\author{
María del Carmen GARCÍA CELA \\ Universidad de Salamanca \\ cela@usal.es
}

Recibido: $20 / 10 / 2012$

Aceptado: 11/01/2013

Résumé

Agota Kristof est née en Hongrie en 1935 mais, forcée à abandonner son pays pour des raisons politiques, elle s'installe en Suisse -à Neuchâtel — en 1956 où elle devient écrivain. En 1986 elle fait paraître Le Grand Cahier, un petit chef-d'œuvre de la littérature contemporaine en langue française, traduit dans plus d'une trentaine de langues. Le grand succès du livre l'encourage à en faire le premier tome d'une trilogie complétée par deux autres volumes, La preuve (1989) et Le troisième mensonge (1991). L'objectif de cet article est d'analyser dans Le Grand Cahier les mécanismes d'énonciation du texte au service de la cruauté.

Mots clés: Littérature Francophone, Suisse, intertextualité, énonciation, répétition, cruauté, sens littéral.

Para una didáctica de la crueldad. El saber-hacer de la escritura en Agota Kristof

\section{Resumen}

Agota Kristof nació en Hungría en 1935 pero, obligada a abandonar su país por razones políticas, se instala en Suiza —en Neuchâtel—- en 1956, donde se convierte en escritora. En 1986 publica El gran cuaderno, una pequeña obra maestra de la literatura contemporánea de lengua francesa, traducida a más de treinta lenguas. El gran éxito del libro la anima a convertirlo en el primer tomo de una trilogía completada por otros dos volúmenes, La prueba (1989) y La tercera mentira (1991). El objetivo de este artículo es analizar en El gran cuaderno los mecanismos de enunciación del texto al servicio de la crueldad.

Palabras clave: Literatura Francófona, Suiza, intertextualidad, enunciación, repetición, crueldad, sentido literal.

\section{For a Didactic of Cruelty. The Know-how of Writing in Agota Kristof}

\begin{abstract}
Agota Kristof was born in Hungary, in 1935 but was forced to abandon her country for political reasons. She settled in Neuchâtel, Switzerland in 1956 where she became a writer. In 1986 she published The Notebook, a little masterpiece of contemporary literature in French, translated into more than 30 languages. The great success of the book encouraged her to turn it into the first tome of a
\end{abstract}


trilogy completed by two other volumes, The Proof (1989) and The Third Ly (1991). The aim of this article is to analyze the textual mechanisms of the enunciation of cruelty in The Notebook.

Key words: Francophone Literature, Switzerland, intertextuality, enunciation, repetition, cruelty, literal meaning.

\section{Referencia normalizada}

García Cela, M. del C. (2013). "Pour une didactique de la cruauté. Le savoir-faire de l'écriture chez Agota Kristof". Thélème, Vol. 28, 141-156.

Sumario: 1. Le Grand Cahier, une histoire cruelle. 2. Principes de vérité et d'objectivité. 3. Siamois énonciatifs. 4. Métabolismes du récit. 5. Poétique du besoin.

Le choix des mots est affaire sérieuse. Il signale toujours une certaine forme d'adoption —ou de refus - des choses, d'intelligence ou de mésintelligence de la réalité.

Clément Rosset, Le choix des mots

Agota Kristof est née en Hongrie en 1935 mais, forcée à abandonner son pays pour des raisons politiques, elle s'installe en Suisse, à Neuchâtel, en 1956. Sa vie est sombre ; son écriture est noire ; toutes deux sont pétries d'ombres et d'exils. " D'un exil l'autre », observe Valérie Petitpierre $(2000,12)^{1}$ dans l'analyse qu'elle lui consacre. Chez Agota Kristof, vie et écriture s'accommodent d'un même biorythme.

Tout aurait pu se passer autrement. Ainsi devait sans doute l'imaginer la petite Agota qui a "attrapé la maladie inguérissable de la lecture » (Kristof, 2004 : 7) dans la salle de cours de son père, qui racontait ses propres histoires à sa grandmère avant de s'endormir et parlait avec son frère une langue inventée. Cependant, la guerre ${ }^{2}$ a éclaté. Envoyée dans un internat, l'adolescente supporte la douleur de la séparation en écrivant ses premiers poèmes, puis représente des sketches tirés de sa plume pour se procurer l'argent qu'elle n'ose demander à sa mère. Après son mariage, elle fuit son pays et finit par arriver en Suisse où elle doit se contenter de travailler dans une usine de montres à Fontainemelon : "À l'usine, tout le monde est gentil avec nous. On nous sourit, on nous parle, mais nous ne comprenons rien » (Kristof, 2004: 42), se souvient-elle. La vie de l'ouvrière sombre dans le silence que viennent bousculer les poèmes en hongrois qui lui trottent dans la tête. C'est pourtant vers le français qu'elle avance, tout en affrontant une autre sorte

\footnotetext{
${ }^{1}$ Pour Valérie Petitpierre, l'exil constitue, chez A. Kristof, un principe d'écriture : «Exilée de son pays, exilée de sa langue maternelle, exilée de son sexe (elle s'est transformée en garçon pour écrire), elle s'exilerait encore de ses textes » (Petitpierre, 2000, 12).

${ }^{2}$ La Hongrie a été envahie par l'Allemagne en 1944 puis par la Russie entre 1944 et 1945.
} 
d'exil non moins poignant: adulte, la lectrice précoce de l'enfance butte sur les signes opaques de la nouvelle langue et demeure en manque de lecture des années durant. Il lui faudra patienter pour que les institutions de la Suisse lui accordent une bourse devant lui permettre de sortir de son illettrisme.

Agota Kristof finira par embrasser le français, non sans déchirements : de toute évidence, sa langue d'adoption ouvre devant elle des horizons jusque là inexistants ; mais il s'agit aussi bien d'une "langue ennemie » (Kristof, $2004: 24$ ) qui empiète sur sa langue maternelle. Établie en Suisse, elle continuera à vivre dans la nostalgie de la Hongrie et du hongrois, puisqu'elle écrit en français, cette langue qu'elle a considérée jusqu'à sa mort comme un territoire à conquérir ${ }^{3}$, cette langue dans laquelle elle s'est toujours dite « analphabète $»^{4}$.

Parler, lire, écrire, telles semblent être les étapes du pèlerinage vers l'écriture. Agota Kristof a dû refaire une deuxième fois le chemin. C'est ce qui explique la publication tardive de son premier roman, Le Grand Cahier —en 1986-, Prix Européen de l'Adelf, traduit dans plus d'une trentaine de langues, adapté à plusieurs reprises au cinéma et au théâtre ${ }^{5}$. Le succès fulgurant du livre l'encouragea à en faire le premier tome d'une trilogie complétée par deux autres volumes, La preuve (1989) et Le troisième mensonge (1991) ${ }^{6}$. Suivirent un quatrième roman, Hier (1995) ; quatre pièces de théâtre rassemblées dans L'Heure grise et autres pièces ${ }^{7}$ (1998); L'Analphabète (2004), où l'autobiographie donne sa réplique à la fiction ; C'est égal (2005), un recueil de récits et, plus tard, Où es-tu Mathias? (2006) ${ }^{8}$ et Le monstre et autres pièces (2007). La marque de l'exil n'a cessé de s'investir dans chacun de ces volumes rompus à la perte.

\footnotetext{
${ }^{3}$ «C'est ainsi que, à l'âge de vingt et un an, à mon arrivée en Suisse, et tout à fait par hasard dans une ville où l'on parle le français, $\mathrm{j}$ 'affronte une langue pour moi totalement inconnue. C'est ici que commence ma lutte pour conquérir cette langue, une lutte longue et acharnée qui durera toute ma vie » (Kristof, $2004: 23$ ).

${ }^{4}$ « Je sais que je n'écrirai jamais le français comme l'écrivent les écrivains français de naissance, mais je l'écrirai comme je le peux, du mieux que je le peux. [...] Écrire en français, j’y suis obligée. C'est un défi. Le défi d'une analphabète » (Kristof, 2004 : 54-55).

${ }^{5}$ A. Kristof pense d'abord donner le livre à L'âge d'Homme, mais on lui conseille d'aller voir les trois grands éditeurs à Paris. Gallimard et Grasset refusent Le Grand Cahier et c'est Seuil qui l'accepte. Trois ans plus tard, Le Grand Cahier est traduit dans 18 langues.

${ }^{6}$ Pour lequel lui est décerné le Prix Goottfried Keller en 2001.

${ }^{7}$ John et Joe (1972), La Clé de l'ascenseur (1977), Un rat qui passe (1972) et L'Heure grise ou le dernier client (1972).

${ }^{8}$ Un récit court suivi d'une pièce de théâtre, Line. Où es-tu Mathias? et Les Heures grises... reprennent des textes écrits pendant les années 70 .
} 


\section{Le Grand Cahier : une histoire cruelle}

Pour le grand public, Agota Kristof prend la parole avec Le Grand Cahier, un récit dont l'action se déroule dans un pays en guerre. Peu importent les précisions géographiques ou temporelles : ce qui compte c'est la cruauté de la guerre.

Dans Le Grand Cahier, A. Kristof compose une intrigue d'allure classique. Une mère de famille vient laisser ses deux garçons, des jumeaux, chez leur grand-mère. Il ne s'agit pas d'un abandon : la campagne est plus sûre que la ville, surtout en l'absence du père, parti en combat. La séparation ne sera pas définitive puisque la famille compte pouvoir se retrouver à la fin de la guerre. Valise en main, les deux jumeaux sont accueillis par la voix sans visage de la grand-mère, appelée dans le coin La Sorcière, alors que la mère quitte les lieux sur la promesse de leur écrire et de revenir un jour les chercher. Et déjà les jeux sont faits : la voix tranchante commence à couper le lien entre la mère et ses enfants à qui on demandera de travailler pour acquitter la dette entamée. « Je vous apprendrai à vivre, moi !» (Kristof, 1986 : 9), dit cette voix.

Malgré tous ces préparatifs, le roman ne se laisse pas aller au mélodrame larmoyant. La guerre et la détresse des jumeaux auraient pu prêter leurs services à la création d'une histoire touchante, mais l'écriture préfère toucher à d'autres fils en s'impliquant à une trame où la saleté trône en majesté, où la poisse crée un biotope qui fonctionne selon ses propres lois. La saleté détruit l'avant-scène du roman, elle ronge la mémoire d'avant le texte, elle érode progressivement les vestiges de toute vie préalable et finit par s'emparer des jumeaux : à force de fréquenter la saleté « nous sentons mauvais comme notre Grand-Mère (Kristof, 1986: 19), constatent-ils, en faisant preuve d'une sorte d'assimilation à l'aïeule.

Attrapés dans cet univers insalubre, les jumeaux gardent les apparences — car la saleté veille sur son domaine - tout en décidant de mener une vie parallèle dans le galetas de la maison où ils écrivent en cachette. La division stratégique de l'espace protège les enfants : en haut, sous le toit, l'écriture; en bas, dans la cave, la grand-mère analphabète et l'abjection. Grâce à ce partage, ils pourront délimiter une circonscription verbale privée ${ }^{9}$, à l'abri des regards indiscrets et des dangers provenant de territoires menaçants que le texte greffe physiquement sur des langues perçues comme déroutantes, voire déviantes : la langue inconnue, délirante, suppurante d'interrogations, de cris, de rires et de sanglots que la grand-mère, en général peu loquace, profère sous les effets de l'alcool (Kristof, 1986: 13); la langue du pays qu'un militaire ennemi énonce maladroitement à l'infinitif; ou encore les langues étrangères qui se succèdent dans la région au gré des nouvelles invasions... Étrangères, infinitives ou alcoolisées, toutes ces langues couvent le

\footnotetext{
${ }^{9}$ C'est que, pour les jumeaux, comme pour G. Bachelard, « Les mots [...] sont de petites maisons, avec cave et grenier » : la vie du poète consisterait même à "monter et descendre, dans les mots mêmes » (Bachelard, 1961 : 139). Voir aussi Alfaro (2011:293).
} 
péril que le texte devra déloger en se munissant d'une langue propre capable de mesurer ses forces à leur pouvoir dévastateur.

C'est à cette tâche primordiale que vont se consacrer les jumeaux. À l'insu de tous, ils préparent l'avènement d'une autre langue, à l'intérieur de la langue, en menant une enquête auprès des mots aisément assimilable à la quête d'un nouvel espace familial. Après avoir éliminé tous les accès, ils sont les seuls à pouvoir monter au grenier, leur sanctuaire secret, au moyen d'une corde (Kristof, 1986 : 11), autre cordon ombilical, les reliant cette fois au sein de l'écriture : là haut, ils installent les remplaçants prêts à occuper le vide laissé par les parents. À commencer par le « Dictionnaire de notre père», le seul vestige paternel transporté dans les bagages ayant résisté à la traversée. Assumant, pour ainsi dire, les fonctions du père, le Dictionnaire offre le catalogue des mots et dicte la loi : dans le Dictionnaire on peut découvrir des mots nouveaux, des antonymes, des synonymes, leur signification et l'orthographe. De son côté, une Bible repérée par hasard chez la grand-mère, sert à pratiquer la lecture à haute voix, à faire des dictées et à apprendre par cœur des pages entières » (Kristof, 1986: 32) La Bible, avec ses mots assemblés dans une suite narrative, leur inculque, disons maternellement, la logique du discours. Bible et Dictionnaire deviennent ainsi les modèles verbaux sur lesquels se règlent les enfants : géniteurs d'encre, ils détiennent la langue et le discours à l'état brut, les germes préfaçant la naissance de l'écriture tout comme la possibilité de recomposer un noyau familial. En guise d'intertexte parental, le couple Dictionnaire-Bible figure un foyer de fortune permettant aux jumeaux d'échafauder une écriture pour affronter la cruauté du monde au présent, désormais le temps du récit. Suite à une sévère discipline, ils consignent les résultats de leur travail dans un «cahier de composition » qu'ils nomment «Le Grand Cahier». Et la tautologie de présider à ce parcours circulaire où les livres se mordent la queue car Le Grand Cahier d'Agota Kristof c'est bien le «Grand Cahier » que composent ces apprentis écrivains.

\section{Principes de vérité et d'objectivité}

«Le Grand Cahier» rapporte les nombreux exercices que s'imposent les deux frères : «Exercices de jeûne » volontaire afin de surmonter le vertige, la nausée, les crampes dans l'estomac; "Exercices d'endurcissement du corps», pour que le corps devienne insensible aux coups ; «Exercices d'endurcissement de l'esprit» pour que les mots blessants ne fassent plus basculer le corps; «Exercices d'immobilité »; "Exercices de mendicité »... L'activité frénétique des enfants intrigue leur entourage. Interrogés sur les raisons qui les poussent à une telle démarche, ils répondent comme si cela allait de soi : « [C'est] pour nous habituer à la douleur. [...] Nous voulons seulement vaincre la douleur, la chaleur, le froid, la faim, tout ce qui fait mal » (Kristof, 1986 : 91). À cette discipline corporelle que s'infligent les garçons pour contourner la douleur, correspond la rigueur des travaux 
de composition répertoriés. Dans Le Grand Cahier, corps et écriture sont taillés d'un même geste.

La mise en mots des événements n'est pourtant pas une tâche facile si l'on demande à l'écriture de dire vrai. L'observation exhaustive du langage obligerait même à une radicale intervention sur la matière verbale car, pour faire juste, il faut d'abord assainir le discours par l'élimination de certains mots qui escamotent la vérité. Parmi ces mots, il existe une catégorie particulièrement suspecte, celle des adjectifs. La manière d'être ou les qualités exprimées par les adjectifs charrient des jugements subjectifs peu fiables. Les enfants en font le constat: « si nous écrivons : 'L'ordonnance est gentil', cela n'est pas une vérité, parce que l'ordonnance est peut-être capable de méchancetés que nous ignorons. Nous écrivons donc simplement: 'L'ordonnance nous donne des couvertures'» (Kristof, 1986:33). Ou encore : «Il est interdit d'écrire 'La petite ville est belle', car 'la petite ville' peut être belle pour nous et laide pour quelqu'un d'autre » (Ibid.). Les nuances de la qualification représentent un excès de poids dont les énoncés doivent se débarrasser s'ils veulent rester vrais. En se pliant à ces contraintes, le discours aspire à une morphologie plus solide, allégée du fard des adjectifs qui méritent donc d'être exilés du texte.

Le déchiquetage s'applique également à certains éléments du lexique à signification incertaine. L'éthique du vrai demande aussi le bannissement des mots ayant trait aux sentiments comme, en l'occurrence, le verbe « aimer» :

Le mot «aimer » n'est pas un mot sûr — découvrent les jumeaux—, il manque de précision et d'objectivité. "Aimer les noix » et " aimer notre mère », cela ne peut pas vouloir dire la même chose. La première formule désigne un goût agréable dans la bouche, et la deuxième un sentiment. Les mots qui définissent les sentiments sont très vagues; il vaut mieux éviter leur emploi (Kristof, $1986: 33)$.

Les mots qui expriment des sentiments —ambivalents, polysémiques, ambigus - nuisent à l'expression du vrai.

Le Grand Cahier s'adonne à une étonnante pédagogie soumettant l'écriture à un dressage non moins étrange, celui d'ausculter puis de purger dans le discours les segments verbaux susceptibles d'évoquer des sentiments ou des jugements subjectifs $^{10}$. Ainsi émondé, le discours devient une matière parfaitement aseptisée alors que l'écriture, osseuse, glisse vers le minimalisme et, fidèle à la logique grammaticale, elle agence un phrasé sans accrocs à la syntaxe stricte et dépouillée. Car c'est au prix de s'interdire de brandir adjectifs et lexique sentimental que, dans Le Grand Cahier, l'écriture se porte en instruments servant «à la description des objets, des êtres humains et de soi-même, c'est-à-dire la description fidèle des faits » (Kristof,

\footnotetext{
${ }^{10}$ Interrogée sur l'absence de sentiments dans ses livres, A. Kristof avoue à Serge Bimpage : « J'en ai eu assez de choses sensibles. Je voulais être seulement juste. Je me méfie du mensonge des sentiments » (Bimpage, 2001).
} 
1986: 33). Sans ces interventions chirurgicales, les principes de vérité et d'objectivité seraient en échec.

Le principe de vérité objective s'érige ainsi en norme de la méthode de composition envisagée par les jumeaux, une objectivité visant à saisir objets, êtres et événements dans la nudité de leur existence soi-disant réelle, brute. Mais le tri des mots prépare d'autre part l'écriture à l'opération de maîtriser ce même réel pour le neutraliser : le réel est imprévisible, inquiétant, et c'est à l'écriture de faire cesser son débordement déconcertant. Chez Agota Kristof, l'écriture chercherait même à surprendre le réel : à le capturer, à le domestiquer par l'inscription, à le happer ici pour qu'il cesse d'être un ailleurs affligeant. Le réel, agrippé à la lettre, porte l'écriture à amalgamer verbe, vrai et réel en un même mortier, à devenir d'ellemême un réel sans dehors, sans échappatoire.

Par obéissance au principe de vérité, l'écriture du Grand Cahier s'adresse aux objets et s'inocule les qualités matérielles aussi bien des objets que des événements du monde extérieur; et, par observance de ce même principe, elle se nantit de propriétés physiques qu'elle ne possède pas d'emblée. La besogne ne consisterait strictement ni dans une fuite du réel ni dans une mise à l'écart de la douleur, mais plutôt dans une telle approximation au réel que l'écriture finirait par devenir physiquement adéquate à ce réel. L'instrument utilisé à cette fin est la répétition. C'est grâce à la répétition des exercices que la brutalité de l'événement mondain est désarçonnée; c'est encore à travers la répétition que, dans Le Grand Cahier, on prend l'habitude de l'intolérable, au point de pouvoir anticiper l'événement douloureux. D'où que la répétition prenne aussi la dimension d'une répétition au sens théâtral du terme : la mise en page d'une écriture physiquement adéquate au réel est encore une mise en scène préparant aux événements blessants à venir. À force de réitération, à force d'exclure adjectifs et émotions, les mots apprennent à durcir de page en page en pourvoyant l'écriture d'énoncés affermissant progressivement leur matière. Les énoncés, objets indurés du discours, pétrissent une sorte de masse compacte sur laquelle devra rebondir l'insoutenable souffrance. Dans l'acte de transcription des mots, l'écriture cultive son geste impassible; dans la physique toute verbale du rebondissement, elle puise son style.

\section{Siamois énonciatifs}

Une si particulière logique objective n'épargne pas le sujet. Rappelons, avec le Trésor de la Langue Française, que l'objectivité est la « qualité de ce qui existe en soi, indépendamment du sujet pensant ». Les vues objectives et objectales du Grand Cahier pourraient ne pas s'arrêter à la stricte capture du réel mais pointer également vers le sujet des énoncés. Car, qui, si ce n'est le sujet, faut-il protéger de jugements et émotions ? C'est bien à travers ses sentiments et ses jugements que le sujet s'expose, que le sujet peut être ciblé par le regard et les actes d'autrui. Si donc les énoncés durcissent c'est non seulement par souci d'objectivité, mais surtout par besoin de blinder le sujet. 
Dans l'univers du Grand Cahier, l'étalement du sentiment heurte dangereusement le sujet. Ainsi, en va-t-il des mots anciens qui remémorent l'amour de la mère : "Quand nous nous rappelons ces mots - affirment les enfants-, nos yeux se remplissent de larmes. Ces mots, nous devons les oublier, parce que, à présent, personne ne nous dit des mots semblables et parce que le souvenir que nous en avons est une charge trop lourde à porter » (Kristof, 1986: 25). Il s'impose donc d'oublier ces mots douloureux. Or les mots nouveaux que le monde en guerre offre aux jumeaux, à savoir les injures qui prennent la place des mots d'amour, ne font pas mieux. Par chance, il existe des antidotes contre les effets nocifs de ces vocables. Des séances d'une demi-heure d'injures par jour suffisent à en contrecarrer les conséquences fatales : «Nous ne voulons plus rougir ni trembler, nous voulons nous habituer aux injures, aux mots qui blessent. [...] Nous nous disons des mots de plus en plus atroces [...] Nous continuons ainsi jusqu'à ce que les mots n'entrent plus dans notre cerveau, n'entrent même plus dans nos oreilles » (Kristof, 1986 : 24).

Contre l'injure, l'habitude de l'injure ; et, contre cette souffrance, boucher les trous du corps (cerveau, oreilles), annihiler le pouvoir des mots au moyen de la répétition : "À force d'être répétés, les mots perdent peu à peu leur signification et la douleur qu'ils portent en eux s'atténue » (Kristof, $1986: 25$ ). Les enfants constatent ce que la théorie de l'énonciation a bien montré, à savoir que le sens de l'énoncé c'est son sujet ${ }^{11}$, et que c'est précisément ce sujet que l'habitude protège de la violence des mots lorsque la répétition habitue le signifiant du mot à perdre l'habitude de s'accompagner du signifié outrageux. La répétition jugule le signifié sentimental qui meurtrit le sujet et se transforme en dispositif au service de l'anesthésie. Dans la répétition, le texte fabrique, pour les enfants, une nouvelle peau, une peau durcie, résistante à la violence ; un épiderme verbal imperturbable face au déchaînement des corps ; une cuirasse de mots qui savent garder leur calme devant les événements déchirants. Ainsi scellé, le corps du texte devient lui-même un objet coriace, une surface imperméable au malheur, une paroi éjectant toute trace du sujet pour mieux épauler sa mise à l'abri. Car, chez Agota Kristof, la limite vers laquelle se rue l'écriture ne possède pas l'envol de la victoire mais la marge plus étroite de la survie. Et c'est dans cette urgence que devra s'opérer le renversement essentiel par lequel l'écriture imposera au réel sa suprématie.

En ce sens, les avatars biologiques de la gémellité ${ }^{12}$ ont leur utilité. L'on sait qu'il existe en Occident une tradition de méfiance à l'égard de la gémellité remon-

${ }^{11}$ Dans Logique, structure, énonciation. Lectures sur le langage, Oswald Ducrot affirmait : « je proposerai que le sens d'un énoncé consiste en une description, au moins partielle, de sa propre énonciation. Les indications relatives aux sujets communicants et modaux relèvent de cette description, qui signale aussi le responsable de l'énoncé et les points de vue qui y sont exprimés » (Ducrot, $1989: 190)$.

${ }^{12}$ Pour une analyse plus approfondie de la gémellité chez Agota Kristof, voir Alfaro, 2011. 
tant à Aristote. Dans ses Problèmes, Aristote ${ }^{13}$ commence par poser que la gémellité " est chose contre nature " puis se corrige aussitôt en affirmant qu'il s'agirait plutôt de cas " extraordinaire[s], sans être contre nature » dans l'espèce humaine - par définition, unipare-. Dans Traité de la génération des animaux le même sujet est abordé dans un chapitre consacré aux «difformités de tout ordre » où le philosophe tente l'explication —assez obscure - de ce qu'il considère être une irrégularité biologique ${ }^{14}$. Plus tard, la science aura donné des réponses précises à la problématique des jumeaux, sans pour autant réussir à effacer l'étrangeté que suscite souvent leur présence.

Dans Le Grand Cahier, le géniteur biologique était, déjà avant la guerre, déstabilisé par leur trop grande proximité et, souhaitant que chacun ait sa propre vie, s'était décidé à séparer les deux frères à l'école. Mais la séparation leur était devenue intolérable parce que ressentie comme une sorte d'amputation corporelle : « cette distance nous semble monstrueuse, la douleur que nous en éprouvons est insupportable. C'est comme si on nous avait enlevé la moitié de notre corps » (Kristof, 1986 : 27), constatent les jumeaux. L'anomalie vue par le père est aussi entendue par un vendeur de papier à l'écoute de leur énonciation bizarre. Le vendeur «n'aime pas du tout [leur] façon de parler ! » (Kristof, $1986: 30$ ) ils parlent même « trop correctement 》 (Kristof, $1986: 30$ ) et leur donne tout ce dont ils on besoin, pourvu qu'ils s'en aillent. Le langage des enfants est bien normatif, mais leurs mots ne sont pas normaux : quelque chose à l'intérieur résonne comme une menace.

Devant le soupçon qu'éveillent les jumeaux, un policier viendra lui aussi faire l'inspection des lieux, mais passera à côté de leur cahier d'exercices où il aurait pu déchiffrer les clés de l'énigme qu'il cherchait à décrypter : « Nous grimpons dans le galetas à l'aide de la corde. Le policier ouvre le coffre où nous rangeons les objets nécessaires à nos études : Bible, dictionnaire, papier, crayons et le Grand Cahier où tout est écrit. Mais le policier n'est pas venu pour lire» (Kristof, 1986: 116). L'inspecteur fouille le monde pour trouver les signes dont seul le lecteur du " Grand Cahier » peut faire l'approche: seul celui qui ose lire a accès au secret. Ce n'est qu'en lisant que l'on apprend que, dans le galetas, les jumeaux écrivent à deux mains : l'un donne le titre, l'autre écrit ; après quoi, ils échangent les feuilles et se corrigent. Si les exercices sont correctement réalisés, ils les gardent dans le « Grand Cahier ». Si non, ils les jettent (Kristof, $1986: 32-33$ ). De l'addition de leurs efforts résulte un seul texte; de la superposition de leurs deux voix un " Nous", le sujet grammatical sur mesure adapté aux exigences de l'écriture.

${ }^{13}$ Section $10:$ « Explication sommaire de quelques faits naturels », paragraphe 28.

${ }^{14}$ « La cause qui produit les jumeaux - affirme Aristote - est la même qui produit les membres en surnombre » et plus loin «c'est la division du sperme qui produit plusieurs embryons, tout comme dans les cours d'eau il se forme des tourbillons » (Aristote, 1887 : Livre IV, Chapitre IV, paragraphes 18-19). 
Avec La Modification, Michel Butor avait accordé au « Vous » son droit de cité en tête de la narration. A. Kristof, pour sa part, donne la parole au « Nous » qui, dans le texte, ne semble être ni un « Nous » de majesté, ni un pluriel à proprement parler. Le « Nous » du Grand Cahier noue devant le lecteur, son seul témoin, deux voix en un même pronom : en les soudant l'un à l'autre, le pronom " Nous » opère la transformation des deux jumeaux en deux siamois dont l'organe partagé est le texte lui-même, l'écriture se portant garante d'une telle union. C'est bien l'écriture qui lie les deux voix du «Nous » en un unisson sans décalage, en une monodie faisant disparaître toute distance interposée entre le sujet et son ombre, masquant toute fêlure par l'empilement des deux instances fondues et confondues. Et le « Nous » d'inventer le nombre duel des siamois énonciatifs, au même endroit où les règles de composition décèlent le sujet.

Cette union indélébile durera le temps du premier roman. Une fois le parcours d'apprentissage accompli et une fois l'enfance dépassée, ils pourront, chacun de leur côté, poursuivre leur chemin dans La Preuve et Le troisième mensonge, les deux romans qui complètent la trilogie de Kristof. Séparés dans ces deux suites romanesques, les jumeaux chercheront sans cesse l'autre disparu en s'essayant à occuper d'autres lieux de la flexion pronominale. Dans La Preuve, une troisième personne raconte la vie de Lucas en l'attente de Claus, le frère jumeau qui a réussi à s'évader. Lorsque Claus reviendra, il ne trouvera de son frère que le manuscrit. Tandis que dans Le troisième mensonge, les récits de Lucas (ou Lucas Claus) et de Klaus (dont le pseudonyme était Klaus Lucas), sont saisis par deux pronoms « je » qui ne parviendront pas à rééditer le " Nous" du Grand Cahier: Lucas et Claus sont faits d'une même pâte, voire d'une même matière phonique, mais autrement redistribuée par l'anagramme qui empêche le calque et la coïncidence exacte des deux noms, des deux sujets. Dans les deux romans, tout se passe comme si « l'auteur s'appliqu[ait] à 'désécrire' les mots des protagonistes, à leur insu et dans leur dos » (Petitpierre, 2000 : 12), à déconstruire le «Nous » qui, dans Le Grand Cahier, avait permis au jumeaux de partager un supplément de corps pour dresser leurs propres lois, étrangères au reste de l'humanité.

Avant d'être séparés en deux moitiés vouées à l'errance, c'est grâce au « Nous » qu'ils avaient acquis le pouvoir sur le texte. Les opérations menées auprès des énoncés qui donnent des ordres le prouvent bien.

$\mathrm{Au}$ début du roman, la grand-mère ordonne: «Le toit et la nourriture, il faut les mériter » (Kristof, 1986 : 14). Le sujet d'autorité profère l'énoncé en vue d'obtenir une réponse du sujet à qui l'énoncé est adressé. Or ce n'est pas un énoncé qui est attendu du récepteur mais un acte dans lequel le sujet de l'autorité prend la place du sujet récepteur. Connaisseurs de la tactique, les jumeaux commencent par se refuser à obéir puis, forcés par les circonstances, ils décident de faire autrement. Au lieu d'accomplir l'ordre par un seul acte, ils multiplient les actes d'obéissance : « Désormais nous faisons tous les travaux que nous sommes capables de faire » (Kristof, 1986 : 15). Dans cette prolifération inattendue, ils reprennent leur place par rapport aux actes et aux énoncés dont découlent ces actes. Les jumeaux ne travailleront plus pour mériter leur gîte, mais parce que c'est «pénible [...] [de] regarder sans rien 
faire, quelqu'un qui travaille » (Kristof, $1986: 14)$ et que «c'est encore plus pénible, surtout si c'est quelqu'un de vieux » (Ibid.). Ils parviendront ainsi à rendre au « Nous » sa place comme sujet de l'énoncé en dévoilant à la grand-mère les raisons qui les poussent à agir: " Nous avons seulement eu honte de nous-mêmes » (Ibid.). Le « Nous", agent de la prolifération, s'assume à part entière comme sujet du moment où il prend l'initiative des actes et des énoncés : des impératifs autres sont à l'œuvre dans ce surcroît d'obéissance visant à ébranler les rapports de domination et même à renverser les relations hiérarchiques. Et les jumeaux apprennent à construire le piège sentimental où pourrait avoir lieu l'acte de destitution du sujet de l'autorité : «Fils de chienne! Vous voulez dire que vous avez eu pitié de moi ? » (Ibid.), en conclut la grand-mère, qui ne sait que trop que les mots ayant trait aux sentiments subjuguent et soumettent. Plus tard, dans le mirage sentimental s'opérera la défaite du sujet du pouvoir, grâce à quoi non seulement les jumeaux deviendront autonomes mais occuperont à leur tour un certain lieu de puissance.

\section{Métabolismes du récit}

C'est bien vers la conquête de ce lieu de puissance que s'achemine le « Nous » en tête des énoncés du discours. Ces énoncés sont immergés dans une logique narrative telle que tout ce qui rebondit sur leur surface solide est autrement métabolisé par le texte. La fin de chaque chapitre a, par exemple, la double fonction de représenter en même temps une conquête et un défi : la conquête d'incorporer au texte un segment de réel maîtrisé à travers l'exercice de composition; le défi de savoir que dehors continuent à suinter les imprévus qu'il va falloir continuer à affronter. C'est précisément de ces défis que l'écriture tire son élan pour arriver au début du chapitre suivant, et de ce mécanisme de concaténation suivie de catapultage qu'elle obtient sa croissance. L'écriture discourt en avant, sans arrière-pensées et entame une sorte de fonction digestive par laquelle elle assimile, sans exceptions, tous les événements du monde qui se rivent à elle. Puis elle cultive par accumulation une particulière mémoire du texte — les enfants n'oublient jamais rien (Kristof, $1986: 111$ ) - : l'inscription garantit la permanence de tout ce qui est écrit et, par là, l'application inexorable de tous les résultats de l'apprentissage. L'écriture gagne du terrain, elle avance sans révoltes et sans s'affronter au réel tout en tissant autour du "Nous » l'enceinte qui le protège. Et, quand tout semble s'anéantir ou chavirer, seuls l'écriture et son sujet tiennent bon. Désormais, ce n'est plus le réel qui incite l'écriture, mais l'écriture qui commence à mobiliser le monde.

L'inflexion a lieu dans l'extrême rapprochement entre les deux instances du « Nous », entre l'écriture et le monde, car dans l'élimination de cette distance s'abolit l'altérité, remplacée par une ipséité superlative. L'on comprend alors qu'Agota Kristof ait pu envisager l'écriture comme un acte presque « suicidaire »: "L'écriture m'empêche de vivre. Je ne vis pas en dehors de l'écriture », affirme-telle dans un entretien avec Philippe Savary (Savary, 1996). Dans Le Grand Cahier, l'écriture se construit comme un tout autarcique prenant le dessus sur le monde, 
voire même sur la vie. C'est à perfectionner cette méthode pédagogique que s'investissent les deux frères.

Le programme pédagogique dont il s'agit ne peut exister dans les cadres d'enseignement institutionnalisés où l'on n'apprend pas à survivre à la guerre. Dès le début, les jumeaux refusent d'assister à l'école comme les autres enfants (Kristof, 1986 : 28) et, en autodidactes, ils inventent d'eux-mêmes leurs exercices d'écriture et de survie au fur et à mesure que le monde leur propose ses événements incompréhensibles; ils formulent des hypothèses qui seront empiriquement testées car il faut « savoir quel effet ça fait et pour observer la réaction des gens » (Kristof, 1986 : 39 ) ; et quand les causes de l'affliction insistent à perdurer, ils s'adonnent à la répétition qui en annule les effets. La répétition transforme l'exception en une régularité qui automatise l'impassibilité comme réaction en face de l'horreur. Lorsque l'impassibilité devient une loi d'accomplissement presque infaillible, la phase propédeutique est accomplie et le programme peut s'élargir à «des livres d'histoire et des livres de géographie [...] qui racontent des choses vraies, pas des choses inventées » (Kristof, 1986: 86), comme la Bible; aux dictionnaires des langues étrangères qu'ils vont maîtriser sans trop de difficultés; et même à une Encyclopédie complète en plusieurs volumes (Kristof, 1986 : 153) volée dans une librairie abandonnée.

Dans Le Grand Cahier, où les libérateurs sont nombreux à circuler, tout le récit oscille entre la prise de pouvoir et la libération. Il n'y a que les jumeaux qui savent que l'autonomie ne peut être préservée que dans les marges et en absence de tout cadrage institutionnel. S'exclure dans les marges signifie aussi s'affranchir de la violence institutionnalisée : celle de l'État, qui légitime la violence, même si son but principal est la protection des individus ; celle de la famille, qui pratique la violence affective au nom de l'amour; celle de la justice, dont la loi inflige sa violence aux innocents ; celle de la religion, qui fait violence à la morale en rendant indécidable la distinction entre le bien et le mal. C'est dans les marges que l'on échappe à la protection et à la surveillance omniprésente, que l'on fabrique ses propres armes. Les jumeaux ont fait leur choix : « Nous ne sommes protégés par personne. Aussi nous apprenons à nous défendre contre les grands » (Kristof, $1986: 57$ ).

« Nous voulons comprendre» (Kristof, 1986: 110), tel est l'objectif du programme des jumeaux. Mais comprendre quoi ? Et d'ailleurs qu'est-ce que comprendre? Dans Le Grand Cahier, c'est la cruauté qui interpelle, qui demande à être examinée. Mais la compréhension qu'elle sollicite n'est pas de l'ordre de la spéculation intellectuelle; la compréhension de la cruauté ne consiste pas dans un recul de la pensée devant son objet pour engendrer l'argument. L'opération qui engage les jumeaux n'est pas mentale, elle a lieu dans le concret, au ras du corps, au ras des mots. Comprendre : cela voudrait plutôt dire inclure par accumulation, englober, intégrer, saisir la matière du monde, une matière qui, comme le signale Agota Kristof « est là, disponible » (Savary, 1996). Saisir, non par l'entendement mais par l'écriture ; abstraire non pour monter vers le concept, mais pour arracher au monde ses objets et en faire des énoncés du texte. Enfin comprendre, non la cruauté comme idée de l'esprit, mais la cruauté en-tant-qu'acte reprise dans l'acte d'écrire. Chez 
Agota Kristof, l'écriture n'est pas un prétexte à penser, elle est autrement un prétexte à performer l'acte de détournement de la cruauté sans passer par la médiation de la pensée.

Agir, non suite à la pensée, mais suite à l'automatisme. Comment sinon affronter la vision du charnier qui porte à son paroxysme la déshérence devant l'abomination ? Un jour, pendant la nuit éclate une bataille et l'odeur dénonce l'infamie des cadavres calcinés:

Nous descendons du mirador, nous trouvons l'entrée. C'est une grande porte de fer, ouverte. Au-dessus, il est écrit en langue étrangère : « Camp de transit. » Nous entrons.

Les bûchers noirs que nous avons vus d'en haut, ce sont des cadavres calcinés. Certains ont très bien brûlé, il ne reste que les os. D'autres sont à peine noircis. Il y en a beaucoup. Des grands et des petits. Des adultes et des enfants. Nous pensons qu'on les a tués d'abord, puis entassés et arrosés d'essence pour y mettre le feu.

Nous vomissons. Nous sortons du camp en courant. Nous rentrons. Grand-Mère nous appelle pour manger, mais nous vomissons encore (Kristof, 1986 : 144-145).

L'horreur de l'amas de chair brûlée s'empare du corps des enfants qui doivent éjecter cette nourriture malsaine. Acculés à l'extrême limite de l'épouvante, où les impératifs de toute morale sont mis en branle, le seul remède à leur portée reste la discipline de l'écriture.

C'est en pratiquant «L'exercice de cruauté » qu'ils ont acquis, bien avant, la méthode pouvant un jour leur servir, alors même que la transgression de toutes les limites tolérables n'était qu'un pressentiment. Puisque la vision du carnage et de la mort les laisse en proie au basculement, il faut pousser plus loin l'exercice de cruauté grâce auquel ils ont déjà appris à répéter l'acte de tuer pour prendre l'habitude de la mort. Jadis, les larmes de la grand-mère n'ont pas pu éviter qu'ils tuent son plus beau poulet et qu'ils répètent l'action tous les dimanches (Kristof, 1986 : 54-55). Mais l'habitude de la mort n'est pas le désir de la mort. La grandmère se trompait lorsqu'elle croyait deviner une soif sanguinaire chez ses petits-fils : « Non, Grand-Mère — disaient-ils—, justement, nous n'aimons pas ça. C'est pour cette raison que nous devons nous y habituer » (Kristof, $1986: 55$ ) Et la grand-mère de répondre : «Je vois. C'est un nouvel exercice. Vous avez raison. Il faut savoir tuer quand c'est nécessaire » (Kristof, $1986: 55$ ).

Arrivée à son comble, la guerre répand la mort sans merci. Contre l'impuissance à interrompre cette propagation insensée, seule demeure l'habitude de la mort come instrument de survie. Dans Le Grand Cahier, la condition de possibilité de cette habitude d'extermination est liée au besoin de la mort et non au désir de la mort. Le désir est aspiration et dépassement du sujet; le besoin se tient à proximité de son objet. C'est du besoin de survie que s'accommodent l'écriture et les actions. Le désir c'est une autre histoire qui, curieusement, dans Le Grand Cahier s'exprime comme pulsion de mort. Ainsi en va-t-il de l'officier qui, en confessant ses peines d'amour, avoue manquer de courage pour se suicider; ou de la voisine désireuse d'une mort qui lorsqu' " on l'appelle, [...] ne vient jamais " (Kristof, $1986: 156$ ); ou encore de la grand-mère qui leur fournit le poison dont ils devront se servir en cas de nécessité. Les jumeaux rendront «ce petit service » (Kristof, 1986:172) à 
leur grand-mère, « trancheron[t] la gorge d'un coup de rasoir » (Kristof, $1986: 156$ ) à la voisine, puis proposeront en toute franchise à l'officier : "Nous vous tuons si vous le voulez vraiment» (Kristof, 1986 : 97). Devenus experts en besoins, ils pourront même offrir la mort sur demande : "Quand il y aura quelque chose à tuer, il faudra nous appeler. C'est nous qui le ferons » (Kristof, 1986:55). La cruelle didactique à laquelle ils se sont soumis les rend capables d'accomplir une telle mission.

Le don de la mort se multiplie en face des énoncés et des sujets aux prises avec le désarroi, qui demandent la mort. De leur côté, les jumeaux, formés au besoin de survie, sont sourds et aveugles au désir, aux pulsions, et se maintiennent près des mots et de leur matière où ils ne dénichent que ce qu'ils croient être des besoins vrais, objectivement exprimés. Si les mots demandent la mort, la mort adviendra sans égard au sujet, sans remords pour celui qui la donne, comme assouvissement d'un simple besoin.

\section{Poétique du besoin}

L'écriture d'Agota Kristof incline la balance du côté du besoin. Vide de déchirements et de sentiments, de prouesses et d'héroïsme, elle ne laisse aucune place à la tragédie, sans doute parce qu'elle en a aussi pris l'habitude. Saisis par la discipline de la répétition, les mots n'ont pas à éprouver le drame du passage difficile de l'idée à la chose, du projet à l'acte, de la conception à la matérialisation des faits. Simplement parce que la répétition y est l'affaire de la chose, de l'acte et de la matérialisation des faits. Sans pathos, on ne crie plus au scandale: l'écriture parcourt aseptiquement l'abomination et les mots n'ont plus peur.

L'assèchement extrême auquel est soumise l'écriture ne permet aucun envol, aucun détour. Chez Agota Kristof, l'écriture ne peut servir à ses fins que si elle est méthodiquement collée au sens littéral. Le sens littéral y est vautré au ras des mots, de la pure carcasse des énoncés, dénudés de sentiments et de souffrances. La logique du sens littéral a, dans Le Grand Cahier, obligé les énoncés à s'habituer à leur nature d'objets opaques au sujet, à dire vrai dans un sens univoque, à se dépouiller de toute ambiguïté : les énoncés n'y sont pas «insondables », comme l'affirme le curé des «voies du Seigneur» (Kristof, $1986: 110$ ), mais un terrain sûr et fiable, sans mystère, avortant systématiquement toute interprétation autre que celle de la signification au pied de la lettre.

Mais à force de contourner la cruauté, Le Grand Cahier aurait pu engendrer une autre sorte de cruauté : la cruauté qui refuse au texte littéraire la polysémie, le sens figuré et le désir. Serait cruel l'acte de vider le texte des zones poreuses de l'ambiguïté, de l'empêcher d'avoir recours à l'image ou au symbole, de le laisser en manque d'espace métaphorique ou de pouvoir d'évocation. Dans Le Grand Cahier, cette cruelle responsabilité retombe tout entière sur le sens littéral qui agence sa poétique du besoin dans ce que Saussure appelait la concaténation syntagmatique, par contagion et par propagation. C'est en surveillant de près la discipline de la 
ligne horizontale que le sens littéral tient sous contrôle l'anarchie que pourrait créer le désir. Et le sens littéral a d'ailleurs tout intérêt à garder son sérieux puisque le désir mène toujours à la mort, puisque le désir menace la survie qui est l'affaire du besoin : le besoin dont doit se contenter le sens littéral par crainte des extravagances du désir ; le besoin astreint au devoir, qui sacrifie le plaisir. « Nous travaillons, nous étudions, nous faisons des exercices » (Kristof, 1986: 37), posent les jumeaux. L'écriture elle-même ne serait qu'une question de travail et de besoin.

Les interdits qui frustrent le désir et le plaisir déteignent encore sur le jeu: « Nous ne jouons jamais » (Kristof, $1986: 36$ ), soutiennent les enfants. La dépense ludique est évacuée de l'enfance scripturale des jumeaux, de même que le jeu érotique en ce sens où, dans Le Grand Cahier «jouer» désigne par métonymie ${ }^{15}$ l'acte amoureux; et l'on sait que les jumeaux ont de bonne heure débarrassé le roman de toute trace de l'amour. Jouer et jouer à l'amour, c'est dangereux. De l'amour Éros ne retrouvera ici que des épaves et des pratiques perverses: zoophilie, pédérastie, violations, sadisme, masochisme. Éros dévaste les corps qui s'exposent à lui. Par chance, les jumeaux sont blindés par l'écriture qui bouche les trous du corps en faisant par là l'économie de toute volupté, de toute jouissance : l'écriture laisse à la chair ce qui est de la chair et aux mots ce qui est des mots.

Cependant, en ouvrant la porte à la métonymie, Agota Kristof fait une concession à l'interprétation. Par métonymie est aussi divulgué un autre jeu majeur, celui de la lecture. Et le lecteur qui joue à lire Le Grand Cahier est pris d'hilarité devant l'hyperbolique séparation de la chair et des mots, devant ce cocasse degré zéro de signification invariante imposée par le sens littéral. Concernant le sens et le rire Clément Rosset affirmait: «Le rire sanctionne [...] une inanité du sens à tous les niveaux : suggérant que toute direction est vaine et toute signification illusoire. » (Rosset, 1995 : 126-127). C'est par le rire que le lecteur fait éclater l'absolu du sens littéral, par le rire qu'il prend à ses frais le désir, le plaisir et le jeu dont a dû se passer le roman.

Ça rit dans le noir, certes, mais à cette distance, ça ne fait plus mal.

\section{RÉFÉRENCES BIBLIOGRAPHIQUES}

Alfaro Amieiro, M., (2007) "Escribir en la frontera. Exilio y escritura en la trilogía de Agota Kristof: Le Grand cahier, La Preuve, Le Troisième mensonge" in Alfaro, M. et al., Más allá de la frontera: cinco voces para Europa. Madrid, Calambour, pp.19-52.

Alfaro Amieiro, M., (2011) « Gémellité, dédoublement et changement de perspectives dans la trilogie d'Agota Kristof : Le Grand cahier, La Preuve, Le Troisième mensonge » in Çédille. Revista de Estudios Franceses [En Ligne]. Monografía

\footnotetext{
${ }^{15}$ Margarita Alfaro ira jusqu'à poser que l'œuvre entière d'Agota Kristof est construite comme une «métonymie de son auteure » (Cf. Alfaro, $2007: 34)$.
} 
de Çédille, $\mathrm{N}^{\circ} 2, \quad$ pp. 283-306, disponible sur : http://cedille.webs.ull.es/M2/12alfaro.pdf [Dernier accès le 20 octobre 2012]

Aristote, (1887) Traité de la génération des animaux, traduit en français pour la première fois et accompagné de notes perpétuelles par J. Barthélémy-Saint Hilaire, tome I. [En ligne]. Paris, Hachette. Disponible sur : http://remacle.org/ bloodwolf/philosophes/Aristote/generation44.htm [Dernier accès le 20 octobre 2012]

Aristote, (1991) Problèmes, traduit en français pour la première fois et accompagné de notes perpétuelles par J. Barthélémy-Saint Hilaire, tome II [En ligne]. Paris, Hachette. Disponible sur : http://remacle.org/bloodwolf/philosophes/Aristote/pro blemes 10.htm [Dernier accès le 20 octobre 2012]

Bachelard, Gaston (1961), Poétique de l'espace, Paris, PUF, [1 $1^{\mathrm{re}}$ Éd. 1957].

Bimpage, Serge, (2001) « Agota Kristof ou l'amour de la vie jusque dans l'enfer », in Tribune de Genève, 22 octobre. [En ligne] Disponible sur: http://www.culturactif.ch/ecrivains/krisoftdg.htm [Dernier accès le 20 octobre 2012]

Ducrot, O., (1989) Logique, structure, énonciation. Lectures sur le langage. Paris, Minuit.

Kristof, A., (1986) Le Grand Cahier. Paris, Seuil.

Kristof, A., (1988) La Preuve. Paris, Seuil.

Kristof, A., (1991) Le troisième mensonge. Paris, Seuil.

Kristof, A., (2004) L'Analphabète Récit autobiographique. Genève, Éditions Zoé.

Petitpierre, V., (2000) D'un exil l'autre: Les détours de l'écriture dans la trilogie romanesque d'Agota Kristof. Genève, Éditions Zoé.

Rosset, C., (1995), Le choix des mots. Paris, Minuit.

Savary, Ph. (1996) "«Écrire c'est presque suicidaire», entretien avec Agota Kristof" in Le Matricule des Anges [En ligne]. $\mathrm{N}^{\circ}$ 14, 1996, disponible sur: http://www.lmda.net/din/tit_lmda.php?Id=3813 [Dernier accès le 20 octobre 2012]. 